\title{
N-(4-metilfenil)-N-(5-(3-hidroksinaftil-2-il)-1,3,4-oksadiazol-2-il) amin'in Sentezi Karakterizasyonu ve Gaussian Hesaplamaları
}

\author{
Murat GENÇ ${ }^{1}$
}

ÖZET: $\mathrm{Bu}$ çalışmada ilk olarak 3-hidroksi-2-naftoikhidrazit bileşiği kullanılarak 3-hidroksinaftil-2-il-N(4-Metilfenil) hidrazinkarbotiyoamid bileşiği sentezlendi. Elde edilen bileşiğin civa(II)asetat bileşiği ile reaksiyonundan $\mathrm{N}$-(4-metilfenil)-N-(5-(3-hidroksinaftil-2-il)-1,3,4-oksadiazol-2-il) amin sentezlendi. Bileşiklerin yapılarıNMR,FT-IR, elementelanalizile karakterize edildi.Çalışmanın ikinci etabında ise; Gaussian09 hesaplamaları kullanılarak HOMO ve LUMO orbital enerjileri, atomların mulliken yükleri, molekülün aktivitesini belirlemek için HOMO-1 ve LUMO+1 orbital enerjileri araştırıldı. Teorik NMR ve FT-IR hesaplamalarının deneysel değerlerle uyumu araştırıldı.

Anahtar kelimeler: 1,3,4-oksadiazol; DFT

\section{The Synthesis, Characterization and Gaussian Calculations of N-(4-methylphenyl)-N-(5-(3-hydroxynahptyl-2-yl)-1,3,4-oxadiazol- 2-yl)amine}

\begin{abstract}
In this study; firstly, 3-hydroxynahptyl-2-yl-N-(4-Methylphenyl) hydrazincarbotihioamide compound was synthesized by using 3-hydroxy-2-nahptonoic hydrazide compound. N-(4-methylphenyl)-N-(5-(3hydroxynahptyl-2-yl)-1,3,4-oxadiazol-2-yl)amine compound was synthesized by the reaction mercury(II)acetate with the obtained compound. The structures of the compounds were characterized by using NMR, FT-IR, elemental analysis. In the second part of the study; HOMO- LUMO orbital energies, atoms' mulliken charges and HOMO-1 and LUMO+1 orbital energies for molecular activity were investigated by using Gaussian 09 . The compatibility of the theoretical NMR and FT-IR with experimental values were investigated.
\end{abstract}

Keywords: 1,3,4-oxadiazole; DFT

Murat GENÇ (0000-0003-1224-4128), Adıyaman Üniversitesi, Fen Edebiyat Fakültesi, Kimya Bölümü, Adıyaman, Turkey Sorumlu yazar/Corresponding Author: Murat GENÇ, mgenc23@gmail.com 


\section{GİRIŞ}

Oksadiazol kapalı formülü $\mathrm{C}_{2} \mathrm{H}_{3} \mathrm{~N}_{2} \mathrm{O}$ olan aromatik heterosiklik bir halkadır. Oksijen ve azot atomunun konumuna göre $1,2,4,1,2,3,1,3,4$ ve 1,2,5 oksadiazol yapısında bulunabilir. Ancak 1,3,4 ve $1,2,4$ oksadiazoller önemli biyolojik ve kimyasal özelliklerinden dolayı araştırmacılar tarafından daha fazla çalışılmaktadır. (Oliveira et al, 2012)

Yapısında 1,3,4-oksadiazol halkası içeren bileşikler geniş bir biyolojik aktivite spektrumuna sahiptir. Genel olarak; antibakteriyel (Kanthiah et al, 2011; Sridhara et.al 2010; Naveena et al., 2010), antifungal (Jayashankar et al., 2009; Akhter et al. 2011) analjezik (Bharathi et al., 2011), antiinflamatuar, antiviral, antikanser (Akhtar et al, 2010; Rostom et al. 2003), antidiabetik (Shyma et al., 2015) etki gösterdikleri son yıllarda yapılan çalışmalarla ortaya konulmuştur.

Ayrıca 1,3,4- oksadiazol halkası içeren iki bileşik şuan klinik tıpta ilaç olarak kullanılmaktadır. Bunlardan biri Raltegravir antiretroviral ilaç olarak kullanılırken Zibotentan anti kanser ilacı olarak kullanılmaktadır. (Savarino, 2006; James, 2009)

Scifinder (SciFinder Scholar - Chemical Abstracts Service)'da 18 Temmuz 2017 tarihinde yaptığımız aramada 1,3,4-oksadiazoller ile ilgili 2010-2017 yılları arasında 860 çalışma kayıtlıdır. Bu sayı 1,3,4-oksadiazollere olan ilginin artarak devam ettiğgini göstermektedir. Bu çalışmada literatürde önemli bir yere sahip olan 1,3,4-oksadiazolün türevi olan N-(4-metilfenil)-N-(5-(3-hidroksinaftil-2-il)1,3,4-Oksadiazol-2-il)amin' in sentezi literatürde ilk kez gerçekleştirildi. Elde edilen bileşiğin teorik parametreleri Gaussian 09 programı ile yapılarak deneysel ve teorik sonuçlar karşılaştırıldı.

\section{MATERYAL VE YÖNTEM}

Çalışmada kullanılan tüm kimyasallar ithal olarak temin edilip ayrıca saflaştırma işlemine tabi tutulmadan reaksiyonlar gerçekleştirildi. Bileşik 1 literatüre göre sentezlendi. (Dogan et al., 1999)

\section{Fiziksel ölçümler}

Elde edilen ürünlerin IR spektrumları Perkin Elmer Spektrum One (ATR) cihaziyla elementel analiz sonuçları CHNS-932 (LECO) Elementel Analiz Cihazı ile, erime noktaları Stuart SMP 30 cihazıyla, NMR analizleri Bruker $400 \mathrm{MHz}$ NMR spektrometresi ve çözücü olarak DMSO-d ${ }_{6}$ kullanılarak gerçekleştirildi.

$N$-(4-metilfenil)-N-(5-(3-hidroksinaftil-2-il)1,3,4-oksadiazol-2-il)amin'in sentezi (2)

$1 \mathrm{mmol}$ 3-hidroksinaftil-2-il-N-(4-metilfenil) hidrazinkarbotiyoamid bileşiğinin bulunduğu (1) 50 mL'lik deney balonuna $30 \mathrm{~mL}$ etil alkol ve $2 \mathrm{mmol}$ Civa (II) asetat bileşiği ilave edildi. Reaksiyona 4 saat çözücünün kaynama noktasında devam edildi. Çöken madde süzüldü. Süzüntü viskoz olana kadar çözücüsü uzaklaştırıldı. Ürün oda sıcaklığında kurutuldu. Etil alkolde kristallendirildi. (Şekil 1)
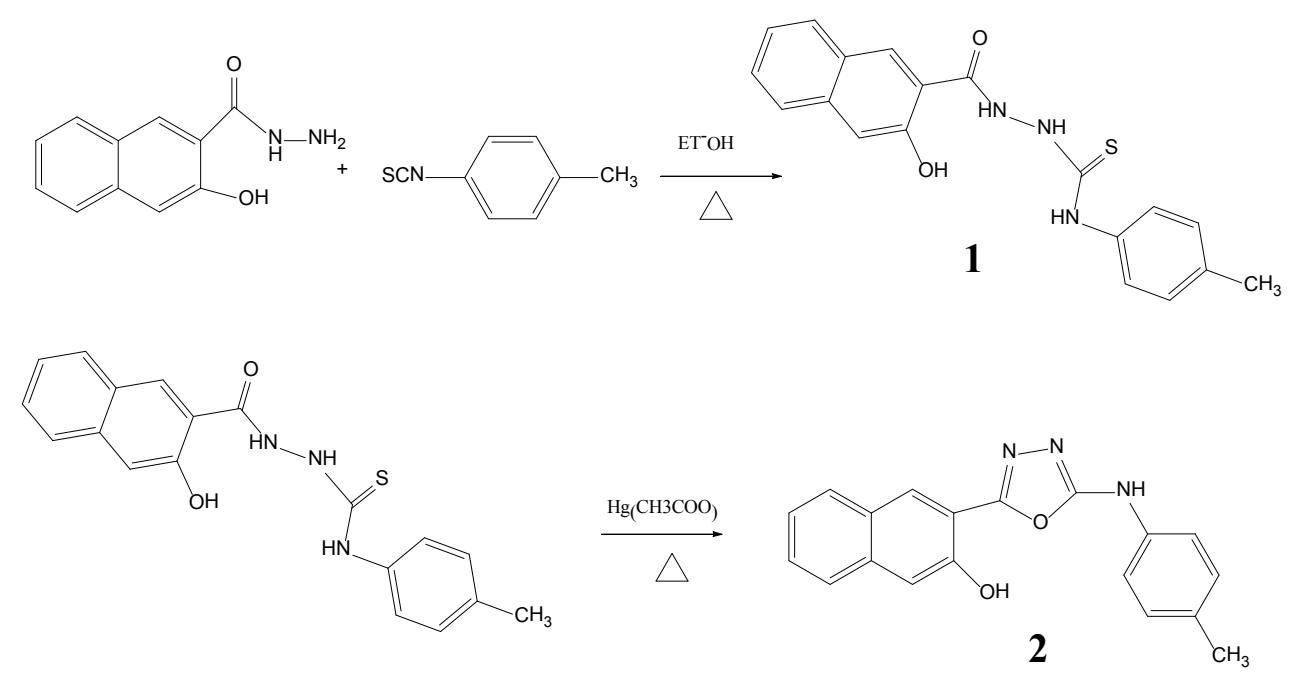

Şekil 1 N-(4-metilfenil)-N-(5-(3-hidroksinaftil-2-il)-1,3,4-oksadiazol-2-il)amin'in sentez şeması 
$N$-(4-metilfenil)-N-(5-(3-hidroksinaftil-2-il)-1,3,4oksadiazol-2-il)amin

Rengi: Beyaz toz, (verim \% 85.) $\left(\mathrm{C}_{18} \mathrm{H}_{13} \mathrm{~N}_{3} \mathrm{O}_{2}\right)$; Elementel Analiz (Hesaplanmış/Bulunan): C: 71.28 (70.92); H: 4.32 (4.16); N: 13.85 (13.56).FT-IR $\left(\mathrm{cm}^{-1}\right)$ : vmax: $3202(\mathrm{OH}), 3239(\mathrm{~N}-\mathrm{H}), 3056-2928-(\mathrm{Ar}-$ $\mathrm{CH}), 2860(\mathrm{C}-\mathrm{H}), \quad 1668,(\mathrm{C}=\mathrm{C}), 1581(\mathrm{C}=\mathrm{N}), 1498$ (N-H), 1188 (C-O). 'H-NMR (400 MHz, DMSO-d6, ppm): $10.69(1 \mathrm{H}, \mathrm{s} ; \mathrm{N}-\mathrm{H}), 10.38(1 \mathrm{H}, \mathrm{s}, \mathrm{OH}), 8.29$ (s 1H, Ar-H), 7.96 (d, 1H, Ar-H, J: 4Hz), 7.78 (s, $1 \mathrm{H}$, Ar-H, j:4Hz), 7.52(m,1H,Ar-H), 7.54(D,2H, Ar-H, J: 8Hz), 7.38(t, 2H, Ar-H, j:8Hz)7.19 (d, 2H, Ar-H, J:8Hz) $2.28\left(3 \mathrm{H}, \mathrm{s}, \mathrm{CH}_{3}\right), 13 \mathrm{C}-\mathrm{NMR}(100$ $\mathrm{MHz}, \quad$ DMSO-d 6 , ppm): $\delta \mathrm{C}=\mathrm{N}: 169, \delta \mathrm{C}=\mathrm{N}: 148$, $\delta_{\text {Aromatik karbonlar: }}: 152,136,135,131,130,128,127,117$, $112,110,112, \delta_{\text {Alifatik karbon }} 20$.
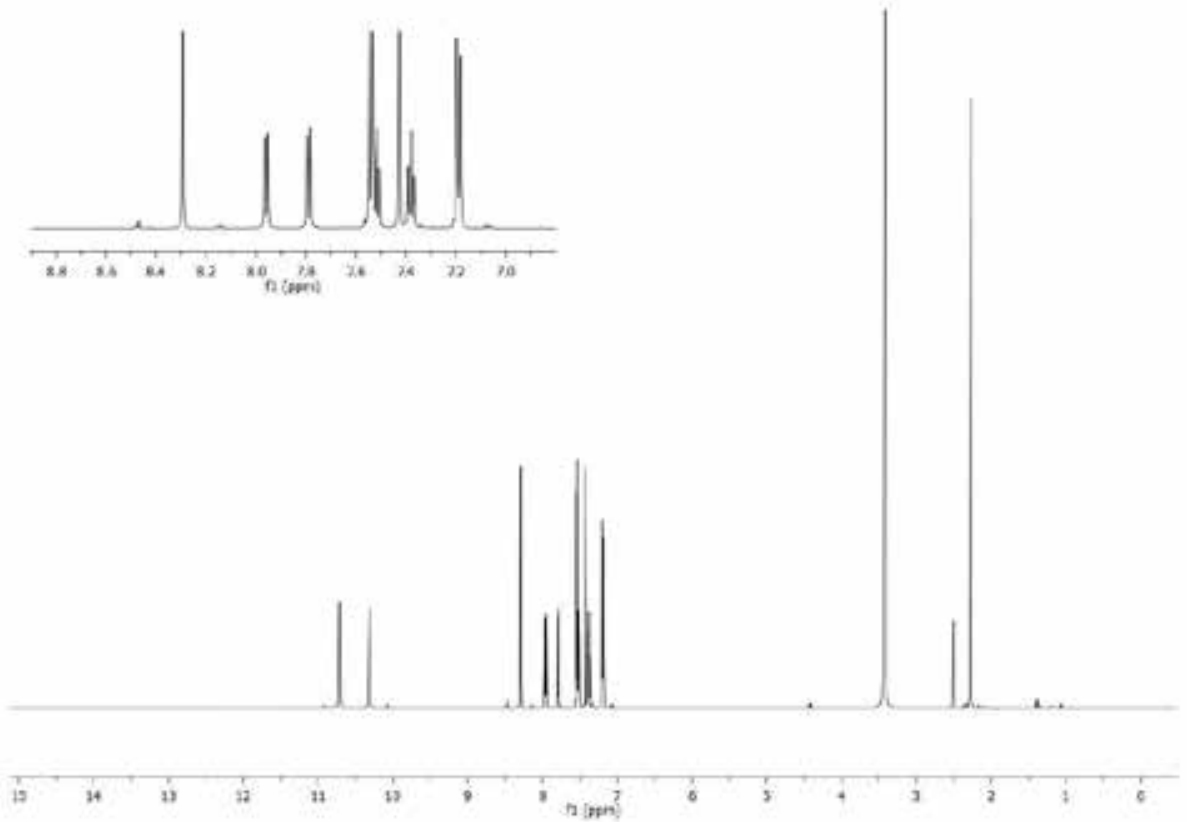

Şekil 2. $N$-(4-metilfenil)-N-(5-(3-hidroksinaftil-2-il)-1,3,4-oksadiazol-2-il)amin' in ${ }^{1} \mathrm{H}-\mathrm{NMR}$ spektrumu

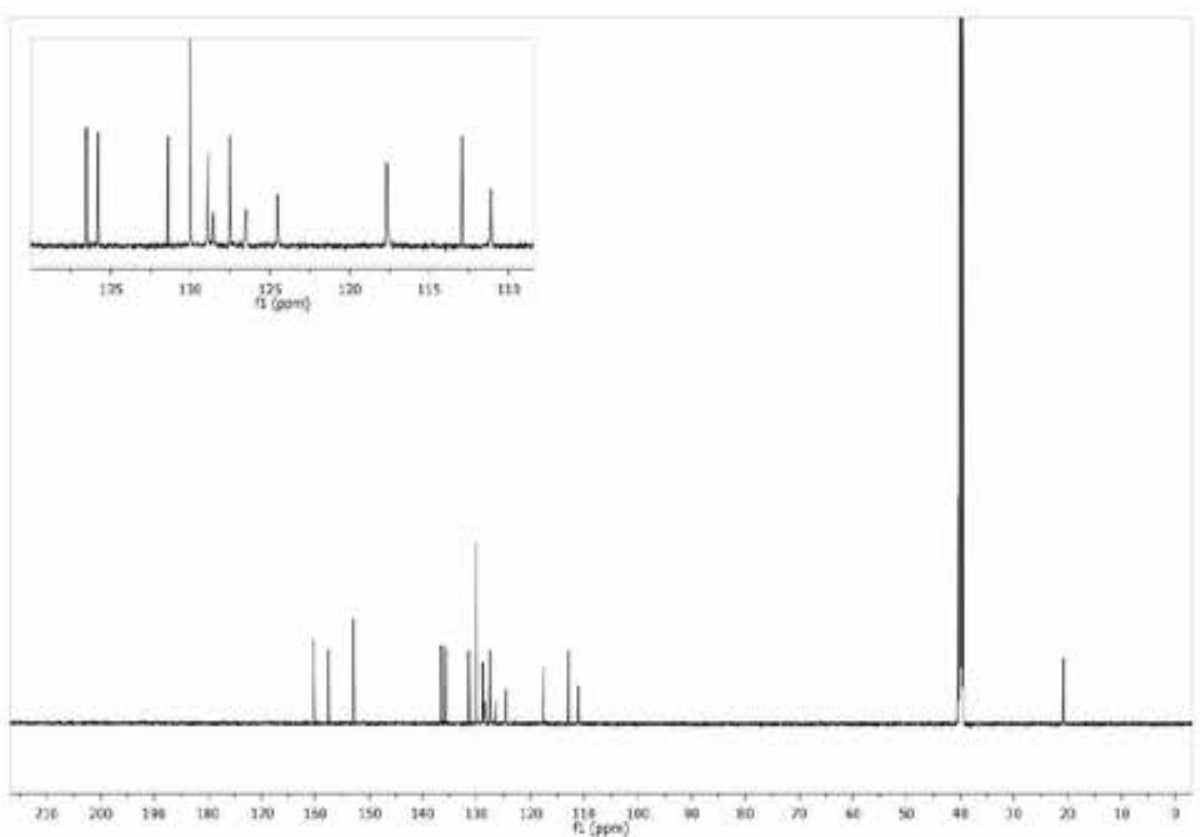

Şekil 3. $N$-(4-metilfenil)-N-(5-(3-hidroksinaftil-2-il)-1,3,4-oksadiazol-2-il)amin' in ${ }^{13} \mathrm{C}-\mathrm{NMR}$ spektrumu. 


\section{Teorik Hesaplamalar}

Teorik hesaplamalar Hp Z820 helios model iş istasyonu kullanılarak Gaussian 09, Gauss view 5.00 programında, DFT $6-311 \mathrm{G}++(\mathrm{d}, \mathrm{p})$ temel seti kullanılarak gerçekleştirildi. Molekülün titreşim frekansları aynı kimyasal modelin gaz fazındaki denge eşitliklerinden hesaplandi.

\section{BULGULAR VE TARTIŞMA}

$\mathrm{Bu}$ çalışmada ilk olarak $N$-(4-metilfenil)- $N$ (5-(3-hidroksinaftil-2-il)-1,3,4-oksadiazol-2-il) amin'in sentezi 3-hidroksi-2-naftoik hidrazit bileşiğinden yola çıkılarak gerçekleştirildi (Şema 1). Elde edilen bileşiğin yapıları FT-IR, NMR ile aydınlatıldı. N-(4-metilfenil)-N-(5-(3- hidroksinaftil-2-il)-1,3,4-oksadiazol-2-il)amin'in sentezinin gerçekleştiğinin en belirgin pikleri FTIR spektrumunda (1) nolu bileşikte görülen 1697 $\mathrm{cm}^{-1}$ 'deki karbonil pikinin kaybolmas1, 3352 ile $3260 \mathrm{~cm}^{-1}$ 'de belirlenen $\mathrm{NH}$ piklerinin tek $\mathrm{NH}$ piki olarak 3239 da gözlemlenmesidir. Ayrıca (1) nolu bileşiğin $\mathrm{C}=\mathrm{N}, \mathrm{C}=\mathrm{C}, \mathrm{C}-\mathrm{H}$ piklerinin sentezlenen (2) nolu bileşikte kayma göstermesi bu varsayımı desteklemektedir. (2) Nolu bileşiğin NMR spektrumu incelendiğinde 10.69 ppm' de bir $\mathrm{NH}$ piki, $10.38 \mathrm{ppm}$ 'de $\mathrm{OH}$ piki, 8.29-7.19 ppm aralığında 10 tane aromatik hidrojen pikleri ile 2.28 ppm'de $\mathrm{CH}_{3}$ piki yapının tam olarak oluştuğunu desteklemektedir..

Çalışmanın ikinci aşamasında elde edilen 1,3,4-oksadiazol bileşiğinin Gaussian 09 programında ilk olarak optimizasyonu gerçekleştirildi.(Şekil 4).

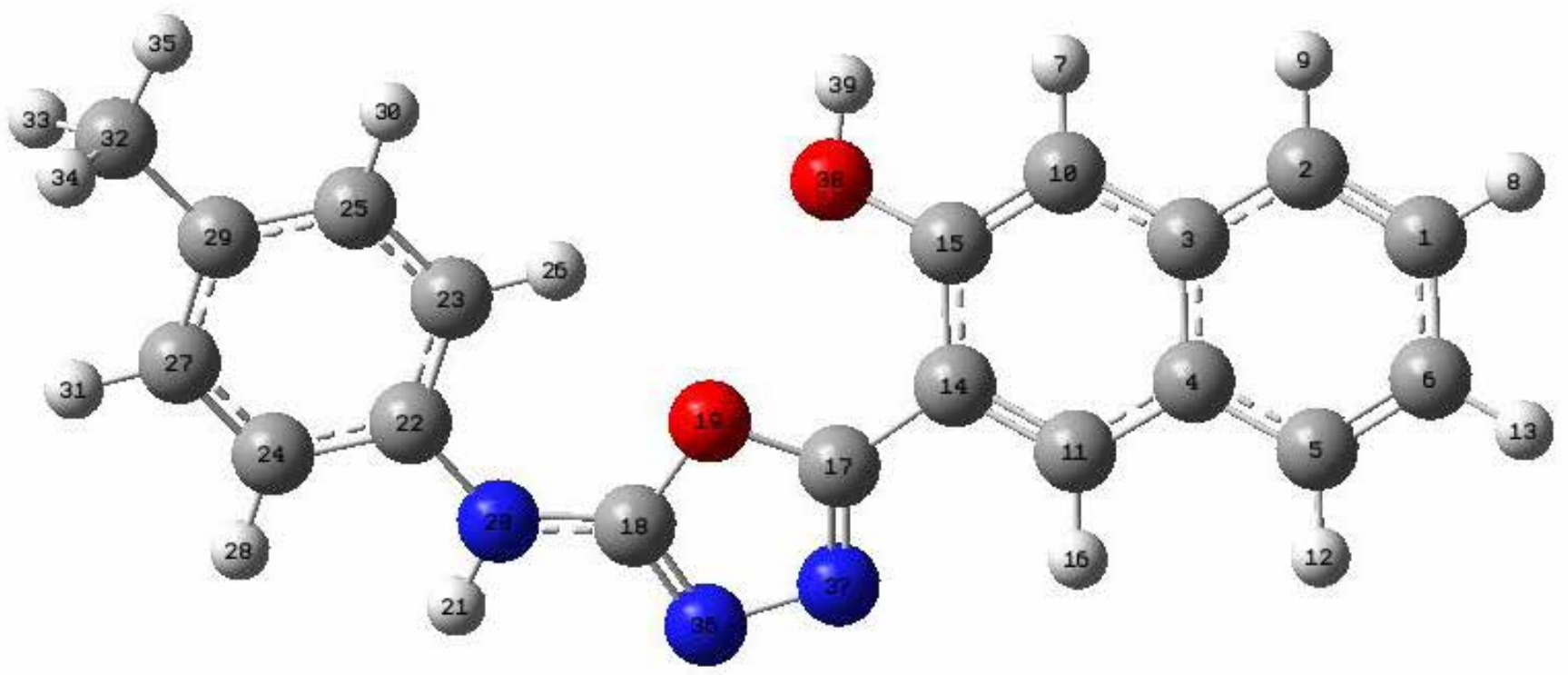

Şekil 4. $N$-(4-metilfenil)-N-(5-(3-hidroksinaftil-2-il)-1,3,4-oksadiazol-2-il)amin'in (2) Gaussian 09' da optimize edilmiş hali

\section{Mulliken Popülasyon Analizi}

Atomik yükler; dipol moment, moleküler polarizasyon, asit-baz davranışı gibi moleküler sistemin pek çok davranışında etkilidir. Bu sebeple Mulliken atomik yük hesaplamaları kuantum kimyasal hesaplamalarında önemli rol oynar. Çalışmada molekülün yük dağılım hesaplamaları
DFT 6-311G++(d,p) temel setinde gerçekleştirilerek Çizelge 1'de listelendi. Buna göre en negatif atom Naftalin halkasına bağlı oksijen olarak gözlemlendi. Diğer heteroatomlarla birlikte genellikle hidrojen bağl1 karbon atomlarıda negatif olarak belirlenirken, en pozitif atom ise oksadiazol halkasındaki 17 numaralı karbon atomu olarak gözlemlendi. 
Çizelge 1. $N$-(4-metilfenil)-N-(5-(3-hidroksinaftil-2-il)-1,3,4-oksadiazol-2-il)amin'nin Mulliken Yük Çizelgesi

\begin{tabular}{lllllllll}
\hline Atom & $\begin{array}{l}\text { Atom } \\
\text { No }\end{array}$ & $\begin{array}{l}\text { Mulliken } \\
\text { Yükler }\end{array}$ & Atom & $\begin{array}{l}\text { Atom } \\
\text { No }\end{array}$ & $\begin{array}{l}\text { Mulliken } \\
\text { Yükler }\end{array}$ & Atom & $\begin{array}{l}\text { Atom } \\
\text { No }\end{array}$ & $\begin{array}{l}\text { Mulliken } \\
\text { Yükler }\end{array}$ \\
\hline $\mathrm{H}$ & 7 & 0.19865 & $\mathrm{C}$ & 18 & 0.68443 & $\mathrm{C}$ & 29 & -0.05445 \\
$\mathrm{H}$ & 8 & 0.20565 & $\mathrm{O}$ & 19 & -0.48263 & $\mathrm{H}$ & 30 & 0.19986 \\
$\mathrm{H}$ & 9 & 0.20213 & $\mathrm{~N}$ & 20 & -0.58902 & $\mathrm{H}$ & 31 & 0.20277 \\
$\mathrm{C}$ & 10 & -0.26386 & $\mathrm{H}$ & 21 & 0.40982 & $\mathrm{C}$ & 32 & -0.58612 \\
$\mathrm{C}$ & 11 & -0.09853 & $\mathrm{C}$ & 22 & 0.15341 & $\mathrm{H}$ & 33 & 0.20877 \\
$\mathrm{H}$ & 12 & 0.20679 & $\mathrm{C}$ & 23 & -0.24056 & $\mathrm{H}$ & 34 & 0.20985 \\
$\mathrm{H}$ & 13 & 0.20627 & $\mathrm{C}$ & 24 & -0.22446 & $\mathrm{H}$ & 35 & 0.20471 \\
$\mathrm{C}$ & 14 & -0.14879 & $\mathrm{C}$ & 25 & -0.17832 & $\mathrm{~N}$ & 36 & -0.37426 \\
$\mathrm{C}$ & 15 & 0.34294 & $\mathrm{H}$ & 26 & 0.23644 & $\mathrm{~N}$ & 37 & -0.28845 \\
$\mathrm{H}$ & 16 & 0.22588 & $\mathrm{C}$ & 27 & -0.18295 & $\mathrm{O}$ & 38 & -0.66770 \\
$\mathrm{C}$ & 17 & 0.47941 & $\mathrm{H}$ & 28 & 0.20021 & $\mathrm{H}$ & 39 & 0.46984 \\
\hline
\end{tabular}

\section{HOMO ve LUMO Analizi}

Moleküler özelliklerin belirlenmesinde nötral bir sistemde En Yüksek İşgal edilmiş Molekül Orbitali (HOMO) ve En Düşük İşgal Edilmemiş Molekül Orbitali (LUMO) anahtar bir rol oynar (Bahgat and Fraihat, 2015). HOMO-LUMO enerji değerleri ve enerji haritaları molekülün kimyasal aktivite ve kinetik kararlılığını temsil eder. Elde edilen bileşiğin HOMO değerleri incelendiğinde elektron yoğunluğunun tüm bileşik üzerinde dağıldığı, LUMO da ise naftalin halkası üzerinde toplandı ̆̆ı belirlendi. Sonuçlar ışında denilebilir ki, yük akışı naftalin halkasına doğrudur.(Şekil 5)

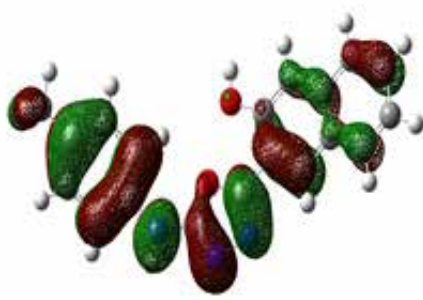

HOMO

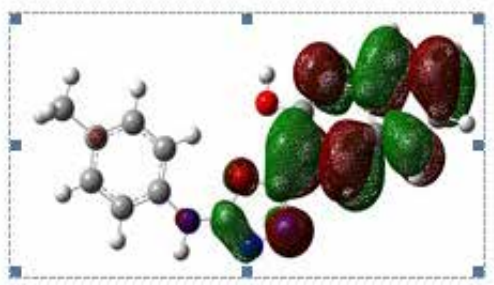

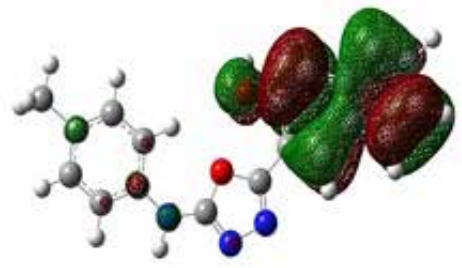

HOMO-1

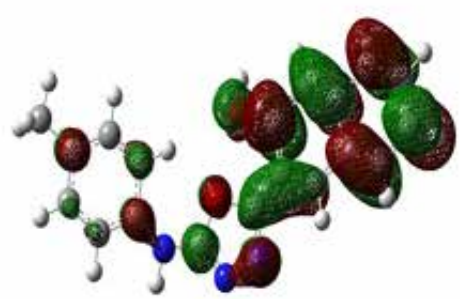

LUMO

$\mathrm{LUMO}+1$

Şekil 5. $N$-(4-metilfenil)-N-(5-(3-hidroksinaftil-2-il)-1,3,4-oksadiazol-2-il)amin' in HOMO-LUMO orbitalleri 


\section{NMR Analizi}

$N$-(4-metilfenil)- $N$-(5-(3-hidroksinaftil-2-il)1,3,4-oksadiazol-2-il)amin'in deneysel NMR analizi DMSO-d 6 çözücü ortamında $400 \mathrm{MHz}$ NMR cihazında, teorik NMR analizi B3LYP/GIAO model setinde alınarak değerler Çizelge 2 ve Çizelge 3' de özetlendi. Deneysel ve teorik NMR değerleri karşılaştırıldığında ${ }^{1} \mathrm{H}-\mathrm{NMR}$ de denklem $\mathrm{y}=0,8963 \mathrm{x}+1,1115 ; \mathrm{R}^{2}=0,6018$ iken ${ }^{13} \mathrm{C}-\mathrm{NMR}$ 'de ise denklem y $=0,9369 \mathrm{x}-3,3219 ; \mathrm{R}^{2}$ $=0,9749$ 'dir. $\mathrm{R}^{2}$ değerleri incelendiğinde ${ }^{13} \mathrm{C}-\mathrm{NMR}$ 'de teorik ve deneysel değerlerde çok iyi bir uyum gözlenirken; ${ }^{1} \mathrm{H}-\mathrm{NMR}$ de bu değer asidik protonların diğer protonlara oranla daha hareketli olmasından dolayı daha düşük çıktığı söylenebilir. Bu protonlar hesaba katılmadı $\breve{g}_{1}$ taktirde $\mathrm{y}=0,9605 \mathrm{x}+0,0803 ; \mathrm{R}^{2}=$ 0,9857 çıkması önerimizi destekler niteliktedir.

Çizelge 2. $N$-(4-metilfenil)-N-(5-(3-hidroksinaftil-2-il)-1,3,4-oksadiazol-2-il)amin' in deneysel ve Teorik ${ }^{1} \mathrm{H}-\mathrm{NMR}$ Değerleri

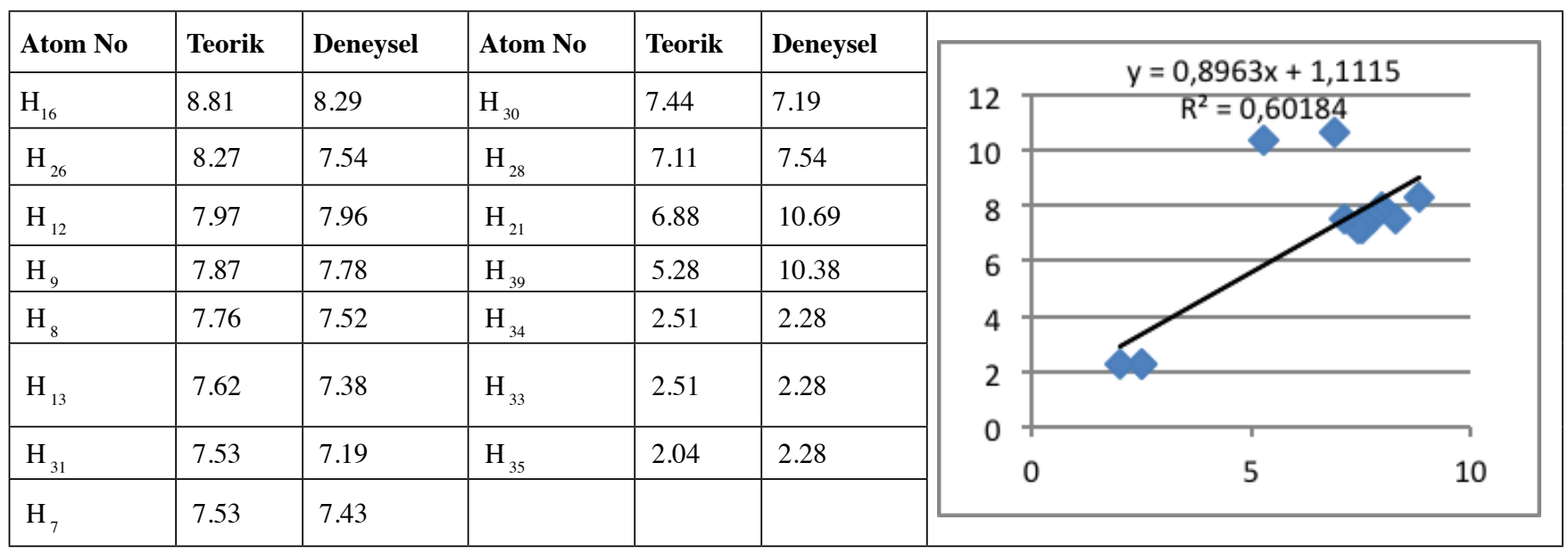

Çizelge 3. N-(4-Metilfenil)-N-(5-(3-Hidroksinaftil-2-il)-1,3,4-Oksadiazol-2-il)amin'in deneysel ve Teorik ${ }^{13} \mathrm{C}-\mathrm{NMR}$ Değerleri

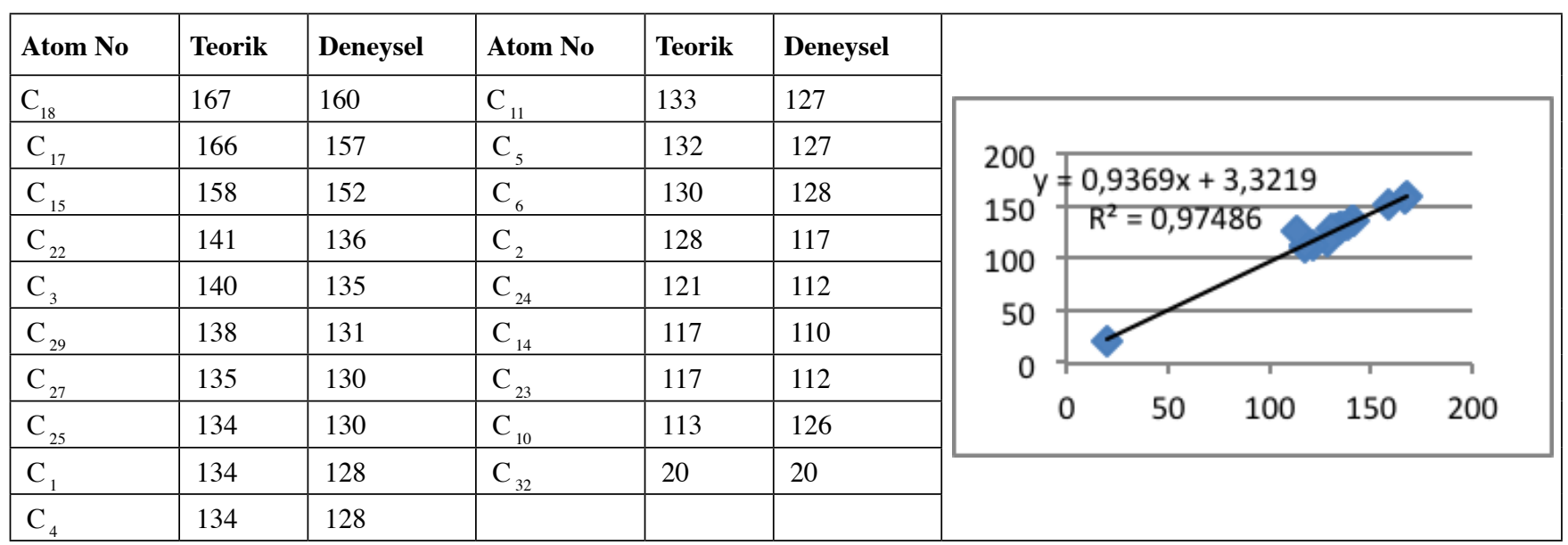

\section{İnfrared Spektrum Analizi}

2 nolu bileşiğin teorik infrared hesaplamaları DFT/B3LYP 6-311 G++(d,p) temel setinde gerçekleştirildi. Gauss-View programı ile İnfrared Bandları görselleştirilerek Çizelge 4'de özetlendi. Elde edilen sonuçlarla deneysel veriler genel olarak uyum içinde olmasına rağmen teorik verilerle deneysel veriler karşılaştırıldığında teorik değerlerin yetersiz kaldı $\breve{g}_{1}$ belirlendi. Bunun temel nedeni deneysel verilerde moleküller arası etkileşimler varken teorik hesaplamaların tek molekül üzerinden gitmesi ve deneysel veriler katı fazda alınırken teorik hesaplamalar gaz fazında alınmasıdır. Deneysel FT-IR sonuçlarında $\mathrm{OH}$ gerilme titreşiminin $3202 \mathrm{~cm}^{-1} \mathrm{de}, \mathrm{NH}$ gerilme titreşimi $3239 \mathrm{~cm}^{-1} \mathrm{de}$, aromatik $\mathrm{CH}$ gerilme titreşimleri 
3056-2928 $\mathrm{cm}^{-1}$ aralığında, $\mathrm{C}=\mathrm{C}$ gerilme titreşimi 1668 $\mathrm{cm}^{-1}$ de, $\mathrm{C}=\mathrm{N} 1581 \mathrm{~cm}^{-1}$ de tespit edilirken; teorik hesaplamalarda ise $\mathrm{OH} 3618 \mathrm{~cm}^{-1}$ de $\mathrm{NH} 3829 \mathrm{~cm}^{-1} \mathrm{de}$,
Aromatik $\mathrm{CH}$ gerilmelerinin $3189-3020 \mathrm{~cm}^{-1}$ aralığında, $\mathrm{C}=\mathrm{C}$ gerilme titreşiminin $1671,1661, \mathrm{C}=\mathrm{N}$ titreşiminin ise $1615 \mathrm{~cm}^{-1}$ de olduğu gözlemlendi.

Çizelge 4. $N$-(4-metilfenil)- $N$-(5-(3-hidroksinaftil-2-il)-1,3,4-oksadiazol-2-il)amin'in hesapsal ve deneysel titreşim frekansları ( $\left.\mathrm{cm}^{-1}\right)$

\begin{tabular}{|c|c|c|c|c|c|}
\hline $\begin{array}{l}\text { Bağ ve titreşim türü } \\
\qquad\left(\mathrm{cm}^{-1}\right)\end{array}$ & 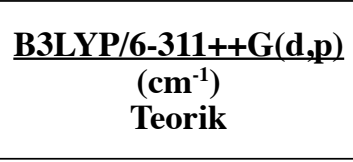 & Deneysel & $\begin{array}{c}\text { Bağ ve titreşim } \\
\text { türü } \\
\left(\mathbf{c m}^{-1}\right)\end{array}$ & $\begin{array}{c}\underline{\text { B3LYP/6- }} \\
\frac{\text { 311++G(d,p) }}{\left(\mathbf{c m}^{-1}\right)} \\
\text { Teorik }\end{array}$ & Deneysel \\
\hline$v(\mathrm{~N}-\mathrm{H})$ & 3829 & 3239 & $\begin{array}{c}\alpha v\left(\mathrm{C}-\mathrm{H}_{2}\right)_{(\text {alifatik })+} \\
v(\mathrm{C}=\mathrm{C})_{(\text {halka) }}\end{array}$ & 536 & 1468 \\
\hline$v(\mathbf{O}-\mathbf{H})$ & 3618 & 3202 & $\omega v\left(\mathrm{C}-\mathrm{H}_{2}\right)_{(\text {alifatik })}$ & 1503 & 1428 \\
\hline $\boldsymbol{v}_{\text {sim }}(\mathrm{C}-\mathrm{H})_{(\mathrm{Ar})}$ & 3189 & 3056 & $\gamma \cup(\mathrm{C}-\mathrm{H})_{(\mathrm{Ar})}$ & 1478 & 1343 \\
\hline$v_{\text {sim }}(\mathrm{C}-\mathrm{H})_{(\mathrm{Ar})}$ & 3159 & 2980 & $\begin{array}{c}v\left(\mathrm{C}=\mathrm{N}_{+} \delta\right. \\
v\left(\mathrm{C}-\mathrm{H}_{2}\right)_{(\text {(alifatik })}\end{array}$ & 1368 & 1318 \\
\hline $\boldsymbol{v}_{\text {asim }}(\mathrm{C}-\mathrm{H})_{(\mathrm{Ar})}$ & 3099 & 2928 & $\begin{array}{c}\mathrm{p} v(\mathrm{~N}-\mathrm{H})+\mathrm{p} v \\
(\mathrm{C}-\mathrm{H})\end{array}$ & 1246 & 1297 \\
\hline $\boldsymbol{v}_{\text {sim }}(\mathrm{C}-\mathrm{H})_{\text {(alifatik) }}$ & 2920 & 2860 & $\mathrm{p} v(\mathrm{~N}-\mathrm{H})$ & 1235 & 1233 \\
\hline$v(\mathbf{C}=\mathbf{C})_{\text {(halka) }}$ & 1671 & 1668 & $v(\mathrm{C}-\mathrm{O})$ & 1188 & 1205 \\
\hline$v(C=C)_{(\text {fenil) }}$ & 1661 & 1639 & tv $\left(\mathrm{C}-\mathrm{H}_{2}\right)$ & 1170 & 1175 \\
\hline$v(C=N)$ & 1615 & 1581 & $\mathrm{p} v(\mathrm{C}-\mathrm{H})$ & 1152 & 1147 \\
\hline$v(\mathrm{~N}-\mathrm{H})$ & 1541 & 1498 & $v(\mathrm{~N}-\mathrm{N})$ & 1098 & 1126 \\
\hline
\end{tabular}

$v$ : gerilme titreşimi; $v_{\mathrm{as}}$ : Asimetrik gerilme titreşimi; $v_{\mathrm{s}}$ : Simetrik gerilme titreşimi; $\omega v$ : Dalgalanma titreşimi; $\vee v$ : Düzlem dışı açı bükülme titreşimi; $\delta v$ : Açı bükülme titreşimi; $p v$ : Sallanma titreşimi.

\section{Moleküler Elektrostatik Potansiyel (MEP) Analizi}

Moleküler Elektrostatik Potansiyel(MEP) molekülde toplam yük dağılımı ile oluşturulan net elektrostatik etki hakkında bilgi vermenin yanı sıra, bir bileşiğin elektronegatifliği, yükü, dipol moment ve kimyasal reaksiyona oranı ile ilişkilendirilirken molekül polaritesini anlayabilmemizi sağlayan optik bir yöntemdir. (Bouabdallah, 2013). Şekil 6'da üç boyutlu olarak elde edilen bileşiğin farklı renklerde MEP haritası gösterilmektedir. Renklerde mavi renk molekülün pozitif bölgelerini gösterirken, yeşil renk ılımlı bölgelerini, kırmızı renk ise negatif bölgelerini gösterir. Buna göre, 2 nolu bileşiğin özellikle oksijen ve azot atomlarının olduğu bölgeler elektronca zenginken, hidrojen atomlarının olduğu yerler pozitif olarak görülmektedir.

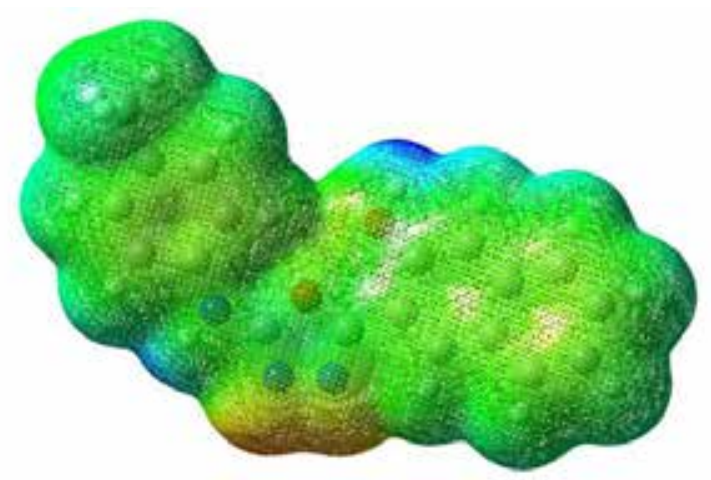

Şekil 6. $N$-(4-metilfenil)-N-(5-(3-hidroksinaftil-2-il)-1,3,4-oksadiazol-2-il)amin bileşiğinin MEP haritası 


\section{Moleküler Parametre Hesaplamaları}

HOMO- LUMO enerjileri, aralarındaki enerji farkları $(\Delta \mathrm{E})$, elektronegatiflik $(\chi)$, kimyasal potansiyel (Pi), kimyasal sertlik $(\eta)$, yumuşaklık $(\sigma)$, elektrik dipol momenti $(\mu \mathrm{M})$, iyonlaşma enerjisi (I), elektron ilgisi (A), elektronik kimyasal potansiyel $(\mu)$ global elektrofillik( $\omega)$ global yumuşaklık, (S) Elektronik Yük ( $\Delta N \max )$ değerleri literatüre göre hesaplandı. (Moser, et al. 2010, Bouabdallah, et al., 2013). Molekülün stabilitesi ve reaktivitesi için kimyasal sertlik ve yumuşaklık önemli parametrelerdir. Sert moleküller büyük enerji değerlerine yumuşak moleküller küçük enerji değerlerine sahiptir. Yumuşak moleküller elektronlarını bir reseptöre kolaylıkla sunabildikleri için sert moleküllerden daha reaktiftir. Diğer moleküler parametrelerde bu değerlerle ilişkilendirilebilmektedir. Buna göre elde edilen bileşiğin HOMO-LUMO değerlerinin düşük olması reaksiyon aktivitesinin daha kolay olacağını ve metal atomları veya bir reseptöre kolayca bağlanabileceğini göstermektedir. Bu nedenle genel olarak 2 nolu bileşiğin aktivitesinin belirlenmesi ve deneysel sonuçların quantum kimyasal hesapları ile ilişkilendirilmesi için yukarıda sayılan parametreler literatüre göre hesaplanarak çizelge 5'de özetlendi.

Çizelge 5. $\mathrm{N}$-(4-metilfenil)-N-(5-(3-hidroksinaftil-2-il)-1,3,4-oksadiazol-2-il)amin bileşiğinin hesaplanan moleküler parametreler Çizelgesi(a.u).

\begin{tabular}{lllc}
\hline HOMO & $-0,20873$ & kimyasal sertlik $(\eta)$ & 0,070005 \\
LUMO & $-0,06872$ & kimyasal yumuşaklık $(\sigma)$ & 14,28469 \\
HOMO-1 & $-0,22808$ & elektronik kimyasal potansiyel (Pi) & $-0,13873$ \\
LUMO+1 & $-0,06365$ & global elektrofillik $(\omega)$ & 0,000674 \\
$\Delta \mathrm{E}_{\text {(номо-LUмO) }}$ & 0,14001 & global yumuşaklık $(\mathrm{S})$ & 0,035003 \\
$\Delta \mathrm{E}_{\text {(номо--LUмO+1) }}$ & 0,16443 & Elektronik Yük $\left(\Delta \mathrm{N}_{\text {max }}\right)$ & 1,981644 \\
elektronegatiflik $(\chi)$ & 0,138725 & & \\
\hline
\end{tabular}

\section{Natural Bă̆ Analizi}

Natural Bağ Analizi molekül içi ve moleküllerarası bağ etkileşimleri için önemli bir parametredir. HOMO iyonizasyon patansiyeli ile ilişkili iken LUMO elektron ilgisi ile ilişkilidir. Çizelge 6'da verilen E2 değeri elektron veren-alan arasındaki etkileşiminin büyüklüğünü göstermektedir. Elde edilen 2 nolu bileşikteki etkileşimleri değerlendirirsek en güçlü etkileşimlerin BD (1) $\mathrm{O}_{38}-\mathrm{H}_{39}$ ile $\mathrm{RY}^{*}(6) \quad \mathrm{H}_{39}$ arasinda $1936.03 \mathrm{kcal} / \mathrm{mol}, \mathrm{LP}$ (1) $\mathrm{O}_{38}$ ile RY*(6) $\mathrm{H}_{39}$ arasinda $1628.53 \mathrm{kcal} / \mathrm{mol}, \mathrm{CR}(1) \mathrm{O}_{38}$ ile $\mathrm{RY}^{*}(6) \mathrm{H}_{39}$ arasında $1213.44 \mathrm{kcal} / \mathrm{mol}$ olduğu gözlemlendi.

Çizelge 6. $N$-(4-metilfenil)-N-(5-(3-hidroksinaftil-2-il)-1,3,4-oksadiazol-2-il)amin bileşiğinin NBO analiz Çizelgesi

\begin{tabular}{|c|c|c|c|c|c|}
\hline Donor NBO & Acceptor NBO & $\mathrm{E}(2) \mathrm{kcal} / \mathrm{mol}$ & Donor NBO & Acceptor NBO & $\mathrm{E}(2) \mathrm{kcal} / \mathrm{mol}$ \\
\hline $\mathrm{BD}$ (1) $\mathrm{C}_{15}-\mathrm{O}_{38}$ & $\mathrm{RY}^{*}(3) \mathrm{H}_{39}$ & 29.54 & $\mathrm{CR}(1) \mathrm{O}_{38}$ & $\mathrm{BD} *(2) \mathrm{C}_{25}-\mathrm{C}_{29}$ & 34.63 \\
\hline $\mathrm{BD}(1) \mathrm{C}_{15}-\mathrm{O}_{38}$ & $\mathrm{RY} *(6) \mathrm{H}_{39}$ & 1082.77 & $\mathrm{CR}(1) \mathrm{O}_{38}$ & $\mathrm{BD}^{*}(1) \mathrm{C}_{32}-\mathrm{H}_{35}$ & 193.02 \\
\hline $\mathrm{BD}$ (1) $\mathrm{C}_{15}-\mathrm{O}_{38}$ & BD*(2) $C_{17}-N_{37}$ & 14.08 & $\mathrm{CR}(1) \mathrm{O}_{38}$ & BD*(1) $N_{36}-N_{37}$ & 599.43 \\
\hline $\mathrm{BD}$ (1) $\mathrm{C}_{15}-\mathrm{O}_{38}$ & $\mathrm{BD}^{*}(2) \mathrm{C}_{25}-\mathrm{C}_{29}$ & 20.12 & $\mathrm{CR}(1) \mathrm{O}_{38}$ & $\mathrm{BD}^{*}(1) \mathrm{O}_{38}-\mathrm{H}_{39}$ & 310.06 \\
\hline $\mathrm{BD}$ (1) $\mathrm{C}_{15}-\mathrm{O}_{38}$ & $\mathrm{BD} *(1) \mathrm{N}_{36}-\mathrm{N}_{37}$ & 159.93 & $\mathrm{LP}(1) \mathrm{O}_{38}$ & $\mathrm{RY}^{*}(3) \mathrm{H}_{39}$ & 30.07 \\
\hline $\mathrm{BD}$ (1) $\mathrm{C}_{15}-\mathrm{O}_{38}$ & $\mathrm{BD} *(1) \mathrm{O}_{38}-\mathrm{H}_{39}$ & 75.74 & $\mathrm{LP}(1) \mathrm{O}_{38}$ & $\mathrm{RY}^{*}(6) \mathrm{H}_{39}$ & 1628.53 \\
\hline $\mathrm{BD}$ (1) $\mathrm{O}_{38}-\mathrm{H}_{39}$ & $\mathrm{RY}^{*}(3) \mathrm{H}_{39}$ & 41.23 & $\mathrm{LP}(1) \mathrm{O}_{38}$ & $\mathrm{BD}^{*}(2) \mathrm{C}_{17}-\mathrm{N}_{37}$ & 22.59 \\
\hline $\mathrm{BD}$ (1) $\mathrm{O}_{38}-\mathrm{H}_{39}$ & $\mathrm{RY} *(6) \mathrm{H}_{39}$ & 1936.03 & $\mathrm{LP}(1) \mathrm{O}_{38}$ & $\mathrm{BD}^{*}(2) \mathrm{C}_{25}-\mathrm{C}_{29}$ & 30.71 \\
\hline $\mathrm{BD}(1) \mathrm{O}_{38}-\mathrm{H}_{39}$ & $\mathrm{BD} *(2) \mathrm{C}_{17}-\mathrm{N}_{37}$ & 25.21 & $\mathrm{LP}(1) \mathrm{O}_{38}$ & $\mathrm{BD}^{*}(1) \mathrm{C}_{32}-\mathrm{H}_{35}$ & 67.72 \\
\hline $\mathrm{BD}$ (1) $\mathrm{O}_{38}-\mathrm{H}_{39}$ & $\mathrm{BD}^{*}(2) \mathrm{C}_{25}-\mathrm{C}_{29}$ & 37.36 & $\mathrm{LP}(1) \mathrm{O}_{38}$ & $\mathrm{BD}^{*}(1) \mathrm{N}_{36}-\mathrm{N}_{37}$ & 122.70 \\
\hline $\mathrm{BD}$ (1) $\mathrm{O}_{38}-\mathrm{H}_{39}$ & $\mathrm{BD} *(1) \mathrm{C}_{32}-\mathrm{H}_{35}$ & 52.38 & $\mathrm{LP}(1) \mathrm{O}_{38}$ & $\mathrm{BD}^{*}(1) \mathrm{O}_{38}-\mathrm{H}_{39}$ & 75.20 \\
\hline $\mathrm{BD}$ (1) $\mathrm{O}_{38}-\mathrm{H}_{39}$ & $\mathrm{BD} *(1) \mathrm{N}_{36}-\mathrm{N}_{37}$ & 212.88 & $\mathrm{LP}(2) \mathrm{O}_{38}$ & $\mathrm{RY}^{*}(1) \mathrm{H}_{39}$ & 36.08 \\
\hline $\mathrm{BD}$ (1) $\mathrm{O}_{38}-\mathrm{H}_{39}$ & $\mathrm{BD} *(1) \mathrm{O}_{38}-\mathrm{H}_{39}$ & 106.73 & $\mathrm{LP}(2) \mathrm{O}_{38}$ & $\mathrm{RY}^{*}(6) \mathrm{H}_{39}$ & 101.04 \\
\hline $\mathrm{CR}(1) \mathrm{O}_{38}$ & $\mathrm{RY}^{*}(15) \mathrm{O}_{38}$ & 30.98 & $\mathrm{LP}(2) \mathrm{O}_{38}$ & $\mathrm{BD} *(2) \mathrm{C}_{10}-\mathrm{C}_{15}$ & 21.92 \\
\hline $\mathrm{CR}(1) \mathrm{O}_{38}$ & $\mathrm{RY} *(16) \mathrm{O}_{38}$ & 33.38 & $\mathrm{BD}^{*}(2) \mathrm{C}_{17}-\mathrm{N}_{37}$ & $\mathrm{BD}^{*}(2) \mathrm{C}_{25}-\mathrm{C}_{29}$ & 11.62 \\
\hline CR (1) $\mathrm{O}_{38}$ & $\mathrm{RY}^{*}(3) \mathrm{H}_{39}$ & 83.73 & $\mathrm{BD}^{*}(2) \mathrm{C}_{22}-\mathrm{C}_{23}$ & $\mathrm{BD}^{*}(2) \mathrm{C}_{24}-\mathrm{C}_{27}$ & 174.20 \\
\hline CR (1) $\mathrm{O}_{38}$ & $\mathrm{RY}^{*}(6) \mathrm{H}_{39}$ & 1213.44 & $\mathrm{BD}^{*}(2) \mathrm{C}_{22}-\mathrm{C}_{23}$ & $. \mathrm{BD}^{*}(2) \mathrm{C}_{25}-\mathrm{C}_{29}$ & 60.20 \\
\hline $\mathrm{CR}(1) \mathrm{O}_{38}$ & $\mathrm{BD} *(2) \mathrm{C}_{17}-\mathrm{N}_{37}$ & 20.15 & $\mathrm{BD}^{*}(2) \mathrm{C}_{24}-\mathrm{C}_{27}$ & . BD*(2) $\mathrm{C}_{25}-\mathrm{C}_{29}$ & 121.21 \\
\hline
\end{tabular}




\section{SONUÇ}

$\mathrm{Bu}$ çalışmada $\mathrm{N}$-(4-metilfenil)-N-(5-(3-hidroksinaftil-2-il)-1,3,4-oksadiazol-2-il)amin bileşiğinin sentezi literatürde ilk kez gerçekleştirildi. Elde edilen bileşiğin yapısı ${ }^{1} \mathrm{H}-\mathrm{NMR},{ }^{13} \mathrm{C}-\mathrm{NMR}$, elementel analiz teknikleriyle aydınlatıldı. Çalışmanın ikinci aşamasında ise Gaussian 09 programıla bileşiğin teorik hesaplamaları yapıldı. Teorik sonuçlara göre HOMO da elektron yoğunluğu tüm bileşiğe dağılmasına rağmen LUMO' da naftalin halkası üzerinde toplandı $\breve{g} 1$, teorik ve deneysel NMR değerleri karşılaştırıldığında $R^{2}$ değerinin ${ }^{1} \mathrm{H}$-NMR'de $0,6018{ }^{13} \mathrm{C}$-NMR'de 0,9749 olarak belirlendi. Deneysel ve teorik FT-IR değerleri genel olarak uyum içerisinde iken, moleküler parametre hesapları sonucunda elde edilen değerlerin düşük olması nedeniyle metal atomlarıyla kolaylıkla bağlanabileceği tespit edildi. Natural bağ analizi sonucuna göre ise en güçlü elektron alan-veren etkileşimlerin $\mathrm{BD}$ (1) $\mathrm{O}_{38}$ $\mathrm{H}_{39}$ ile $\mathrm{RY}^{*}(6) \mathrm{H}_{39}$ arasında $1936.03 \mathrm{kcal} / \mathrm{mol}$ olduğu gözlemlendi.

\section{TEŞEKKÜR}

$\mathrm{Bu}$ çalışma Adıyaman Üniversitesi Bilimsel Araştırma Projeleri Koordinasyon Birimi (Proje No: FEFBAP/2014-0003) tarafından desteklenmiştir.

\section{KAYNAKLAR}

Akhter M, Husain A, Azad B, Ajmal M, 2009. Aroylpropionic acid based 2,5-disubstituted-1,3,4-oxadiazoles: Synthesis and their anti-inflammatory and analgesic activities. European Journal of Medicinal Chemistry , 44: 2372-8.

Akhtar T, Hameed S, Al-Masoudi NA, Loddo R, Colla, PL, 2010. In vitro antitumor and antiviral activities of new benzothiazole and 1,3,4-oxadiazole-2- thione derivatives. Acta Pharmazie, 58: $135-49$

Bharathi D, Hemalatha S, Devadass G, Kumar PR, Shanmugasundaram P, Aanandhi MV, 2010, Synthesis, Characterisation and in-vitro Antiinflammatory and Anthelmintic activities of 1,3,4-Oxadiazole derivatives. International Journal of Chem Tech Research, 2(4): 1867-70.

Bahgat K, Fraihat S, 2015. Normal coordinate analysis, molecular structure, vibrational, electronic spectra and NMR investigation of 4-Amino-3-phenyl-1H-1,2,4-triazole-5(4H)thione by ab initio HF and DFT method. Spectrochimica Acta Part A: Molecular and Biomolecular Spectroscopy,135: 1145-1155.

Bouabdallah I, Rahal M, Harit T, El Hajbi A, Malek F, Eddike D, Tillard M, Ramdani A, 2013. Hartree-Fock and density functional theory studies on tautomerism of 5,5-diisopropyl3,3-bipyrazole in gas phase and solution. Chemical Physics Letters, 588: 208-214

Dogan HN, Duran A,Yemni E, 1999. Synthesis and antibacterial activity of 1-(3-hydroxy-2-naphthoyl)-4-substituted thiosemicarbazides. Drug Metabolism and Drug Interactions, 15: $187-195$

James ND, Growcott JW, 2009. Zibotentan. Drugs Future, 34: 624-633.

Jayashankar B, Rai KML, Baskaran N, Sathish H.S, 2009. Synthesis and pharmacological evaluation of 1,3,4- oxadiazole bearing bis(heterocycle) derivatives as anti-inflammatory and analgesic agents. European Journal of Medicinal Chemistry, 44: 3898-902.
Kanthiah S, Kalusalingam A, Velayutham R, Vimala AT, and Beyatricks J, 2011. 5-(2-aminophenyl)-1,3,4- oxadiazole$2(3 \mathrm{H})$-thione derivatives Synthesis, characterization and antimicrobial evaluation. International Journal of Pharmaceutical Sciences Review and Research, 6(1): 64-7.

Moser A, Range K, York DM, 2010. Accurate Proton Affinity and Gas-Phase Basicity Values for Molecules Important in Biocatalysis. The Journal of Physical Chemistry B, 114: 13911-13921.

Naveena CS, Boja P, Kumari NS, 2010. Synthesis, characterization and antimicrobial activity of some disubstituted 1,3,4-oxadiazoles carrying 2-(aryloxymethyl)phenyl moiety. European Journal of Medicinal Chemistry ,45:4708-19.

Oliveira C, Lira B, Barbosa-Filho J, Lorenzo JGF. and AthaydeFilho P, 2012. Synthetic Approaches and Pharmacological Activity of 1,3,4-Oxadiazoles: A Review of the Literature from 2000-2012. Molecules,17: 10192-10291.

Rostom SAF, Shalaby MA, EIDemellawy MA, 2003. Synthesis of new 1-(4- chlorophenyl)-4-hydroxy-1H-pyrazole3-carboxylic acid hydrazide analogs and some derived ring systems. A novel class of potential antitumor and anti-HCV agents. European Journal of Medicinal Chemistry .38: 959-74.

Savarino A, 2006, A historical sketch of the discovery and development of HIV-1 integrase inhibitors. Expert Opinion on Investigational Drugs, 15: 1507-1522.

Shyma PC, Balakrishna K, Peethambar SK, Vijesh AM, 2015. Synthesis, characterization, antidiabetic and antioxidant activity of 1,3,4-oxadiazole derivatives bearing 6-methyl pyridine moiety, Der Pharma Chemica, 7(12): 137-145

Sridhara AM, Reddy KRV, Keshavayya J, Goud PSK, Somashekar BC, Bose P, 2010. Synthesis and antimicrobial activity of 2-substituted [4-(1,3,4-oxadiazol-2-yl methyl)] phthalazin$1(2 \mathrm{H})$-one derivatives. European Journal of Medicinal Chemistry, 45: 4983-9. 